\title{
Regards sur les forfaits par cas et le financement hospitalier en 2013
}

\section{Dominik Franz, \\ Holger Bunzemeier, \\ Wolfgang Fiori, \\ Norbert Roeder}

DRG-Research-Group, Clinique universitaire de Münster (direction: Prof. N. Roeder)
Correspondance:

Dr Dominik Franz

Secteur management médical controlling médical, DRG-Research-Group Clinique universitaire Domagkstrasse 20 D-48129 Münster Tél. 00492518352023 Fax 00492518352019

dominik.franz[at]ukmuenster.de

\section{Introduction}

Le changement de système dans la rémunération des prestations hospitalières stationnaires a été accompli. La version 1.0 de SwissDRG est en effet, depuis le $1^{\text {er }}$ janvier 2012, le système qui sert de base à l'indemnisation des cas somatiques stationnaires dans les hôpitaux de soins aigus et les maisons de naissance [1]. Mise au point pour l'année 2013, la ver-sion 2.0 a, elle aussi, déjà été publiée [2]. Après de premières expériences dans l'application, les fournisseurs de prestations et les répondants des frais se posent des questions importantes. Avec la qualité actuelle des données et la méthode de calculation des coûts, une rémunération correcte peut-elle être garantie? Comment faut-il traiter les domaines médicaux hautement complexes, dont le nombre de cas est faible? Le catalogue CHOP est-il suffisamment différencié pour fournir déjà des informations sur les ressources pour la calculation des coûts et le regroupement des cas?

Le présent article décrit les nouveaux développements importants de la version 2.0 de SwissDRG et signale également les problèmes encore à résoudre.

\section{La version 2.0 de SwissDRG}

La version de planification 2012/13 de la version 2.0 a été publiée en août 2012 [2]. Elle permet un groupage des données de l'année 2012. Lors de sa conception, SwissDRG SA a encore pu profiter des expériences réalisées par l'Institut DRG allemand InEK. Le tableau 1 présente les versions 1.0 et 2.0.

\section{Classification des diagnostics et des procédures}

Pour la collecte des données 2012, on s'est basé sur la CIM-10-GM, version 2010, et sur la CHOP, version 2012. Parmi les changements importants entre la CIM-10-GM, versions 2009 et 2010, et la version 2008, il y a par ex. l'introduction de nouveaux codes pour le syndrome du pied diabétique et les réarrangements de codes CIM pour l'appendicite aiguë, pour les entérites chroniques, les ulcères de décubitus ainsi que l'insuffisance rénale chronique.

Les résultats de la procédure de propositions de SwissDRG 2010 sont à l'origine du passage de la CHOP 2011 à la version de 2012. Les nouveaux codes CHOP se rapportent par exemple aux opérations de chirurgie vasculaire et thoracique, aux interventions

\section{Résumé}

Le passage au système SwissDRG a maintenant eu lieu. Et la version $\mathbf{2 . 0}$ de SwissDRG, qui sera valable dès 2013, est déjà prête. Le nombre de DRG y a été réduit de 61 unités. Cette réduction concerne surtout les cas hautement complexes des domaines de la respiration artificielle de longue durée, de la néonatologie et de l'oncologie. La version 2.0 améliore, dans la limite des possibilités existantes, la justesse de la prise en compte des prestations par rapport à la version précédente. Néanmoins, la qualité du système atteinte jusqu'ici peut encore être optimisée; elle n'est pas encore suffisante dans la version 2.0 non plus, pour calculer les prestations hospitalières de façon correcte sur la base des données suisses. La version 2.0 ne représente qu'une étape intermédiaire sur la voie visant à atteindre l'objectif prescrit par le législateur pour l'introduction de SwissDRG. La prochaine version (3.0) se fondera notamment sur des changements majeurs des structures CIM-10-GM et CHOP, qui offriront des possibilités de classification plus différenciées du codage des diagnostics et des procédures, si bien qu'on pourra s'attendre à l'avenir à une nouvelle augmentation de la justesse de prise en compte des prestations.

cardiologiques, aux procédés d'imagerie ainsi qu'à l'extension des traitements complexes.

Les changements de la CIM-10-GM et de la $\mathrm{CHOP}$ offriront des possibilités de classification plus différenciées du codage des diagnostics et des procédures. Notamment pour la CHOP, il en résulte, par ex. pour les procédures de chirurgie vasculaire, une présentation plus spécifique de l'utilisation des ressources [3]. Néanmoins, les codes CHOP ne permettront toujours pas de coder par ex. des médicaments coûteux au cas par cas, si bien que des listes séparées devront compléter les saisies (voir ci-après). 
La structure tarifaire de SwissDRG version 2.0

La version 2.0 contient 61 forfaits par cas de moins que la version précédente. Cette diminution concerne exclusivement des DRG tarifés, notamment dans les catégories majeures de diagnostics (MDC) de la pré-MDC (cas hautement complexes avec transplantation ou respiration artificielle de longue durée), de la MDC15 nouveau-nés et de la MDC17 néoplasies hématologiques et solides (tableau 1).

A titre d'exemple, nous prendrons ici la diminution du nombre de DRG dans la pré-MDC. Celle-ci comprend des groupes de cas cliniquement et économiquement très complexes, mais dont le nombre est très petit (par ex. respirations artificielles de longue durée $>1000$ h). Une calculation statistiquement supportable sur la base des données suisses est rendue difficile par le faible nombre de cas. A l'avenir également, on ne s'attend pas à une augmentation du nombre de cas [4]. Or, avec un échantillon trop petit, le risque existe de voir apparaître des coûts relatifs à fortes variations, ne correspondant pas aux coûts moyens effectifs du pays pour l'année de facturation $[5,6]$. Pour la version 1.0 , on peut admettre que la majeure partie des coûts relatifs dans la pré-MDC et la MDC15 ont été établis sur la base de coûts relatifs allemands helvétisés.

Dans la version 2.0, l'échelonnement des durées de respiration artificielle prises en compte par les DRG a été réduit. Il s'ensuit une diminution de l'étendue de tarification et de la hauteur des coûts relatifs dans la pré-MDC. Une mesure comparable a été mise en ouvre pour les nouveau-nés avec un poids faible à la naissance $(<1000$ g). Pour les hôpitaux spécialisés dans les cas de traitement hautement complexes (par ex. les hôpitaux universitaires), il peut aussi y avoir une réduction du case-mix et, suivant la situation des coûts et la valeur du taux de base, un sous-financement. Toutefois, la condensation de DRG augmente le nombre de cas dans les DRG restants, de telle sorte que les conditions pour une calculation des coûts fondée uniquement sur les

\begin{tabular}{|c|c|c|}
\hline \multicolumn{3}{|c|}{$\begin{array}{l}\text { Tableau } 1 \\
\text { Comparaison de versions de SwissDRG [4]. } \\
\text { Remarque: les données sur les coûts relatifs helvétisés et ceux calculés sur la base des données } \\
\text { suisses ont été repris d'une diapositive de SwissDRG SA. Les deux indications pour 2012/13 } \\
\text { ont été reprises bien que la somme des deux chiffres ne donne pas } 975 \text {. }\end{array}$} \\
\hline & Version 1.0 & Version 2.0 \\
\hline n DRG & 1.052 & $991(-5,8 \%)$ \\
\hline n DRG tarifés & 1.036 & $975(-5,9 \%)$ \\
\hline n DRG non tarifés & 16 & $16( \pm 0)$ \\
\hline $\begin{array}{l}\text { n DRG calculés sur la base de } \\
\text { données suisses }\end{array}$ & 804 & 812 \\
\hline $\mathrm{n}$ coûts relatifs helvétisés & 232 & 161 \\
\hline Rémunérations supplémentaires (RS) & Dans les deu & RS tarifées et 2 non tarifées \\
\hline
\end{tabular}

données suisses s'améliorent (tableau 1), ce qui n'est pas sans intérêt, car les structures de soins divergent par rapport à l'Allemagne [4].

Entre les deux versions de SwissDRG, il n'y a pas eu de changement concernant les rémunérations supplémentaires, à savoir: trois tarifées (diverses dialyses) et deux non tarifées («cœur artificiel» et facteurs de coagulation chez les patients hémophiles). Les rémunérations supplémentaires peuvent être facturées en plus d'un forfait SwissDRG et indemnisent des coûts extrêmement élevés pour des traitements déterminés qui ne peuvent être limités à des DRG particuliers. Les rémunérations supplémentaires tarifées le sont avec un montant en francs, unique pour toute la Suisse. Quant aux rémunérations supplémentaires non tarifées, le montant d'indemnisation doit être négocié par chaque hôpital [4]. La version 2.0, elle non plus, ne prévoit pas de rémunérations supplémentaires pour les médicaments coûteux (par ex. les anticorps, les antimycotiques, etc.) ou les produits médicaux onéreux (par ex. implants spéciaux, prothèses, etc.), ce qui peut s'avérer problématique pour des constellations de cas complexes, notamment en oncologie, rhumatologie, orthopédie et chirurgie des accidents, chirurgie vasculaire et neurologie. Dans ces domaines, des médicaments ou des implants coûteux peuvent aboutir à une hétérogénéité considérable des prestations et des coûts, laquelle ne peut pas être prise en compte de manière correcte uniquement par les DRG (voir ci-après). Il en résulte une incitation économique à poser des indications restrictives ou à donner la priorité à des produits de moins bonne qualité dans les cas d'implants.

Hormis les DRG et les rémunérations supplémentaires, l'indemnisation des innovations constitue aussi un élément important du financement hospitalier, que nous n'allons toutefois pas approfondir dans cet article. La procédure présentée par SwissDRG SA pour le financement des innovations permet à chaque hôpital d'entamer des négociations très tôt, mais prévoit une période d'au moins 5 ans pour la mise en application.

\section{La calculation des coûts}

Des cas de traitement dont la complexité diffère ne sont pris en compte dans un système DRG que si les coûts sont calculés correctement dans les hôpitaux. Cela vaut notamment pour l'attribution au cas par cas des coûts élevés particuliers [5, 6]. Dans la version 2.0 non plus, la qualité de la calculation des coûts par cas n'est pas encore suffisante pour calculer les prestations dans les hôpitaux correctement et seulement sur la base des données suisses [4].

Afin d'augmenter la qualité des données, on a unifié, pour le relevé 2012, les listes relatives aux médicaments, procédés et implants coûteux à saisir dans la statistique médicale et on a défini un seuil minimal pour les médicaments onéreux [7]. Pour le 
relevé des données 2013, il est prévu de faire une distinction entre les centres de charges «salle d'op» et «anesthésie» [4]; et dès le relevé 2014, le système REKOLE $^{\circledast}$ [8] deviendra obligatoire comme méthode unique pour l'établissement de la comptabilité analytique [7]. Ce sont là des étapes importantes pour permettre des calculations correctes sur la base des données suisses.

Ces changements sont nécessaires, car plus le système SwissDRG se développera de façon autonome et plus le recours à des données de coûts allemandes helvétisées deviendra problématique sur le plan méthodique $[5,6]$. Vu notamment la participation obligatoire au relevé de 2013 de tous les hôpitaux de soins aigus suisses, avec leurs données de coûts, SwissDRG SA se retrouvera face au défi majeur de devoir permettre aux hôpitaux de participer à la calculation malgré des directives de calculation plus strictes. Or, les expériences allemandes montrent que la calculation de prestations médicales complexes demande un temps considérable et des collaborateurs hospitaliers expérimentés [5, 6]. Le recensement complet augmente certes le nombre de cas, mais une qualité insuffisante des données peut influencer négativement la qualité de la calculation.

\section{Les taux de base individuels}

Grâce à la négociation de taux individuels pour chaque hôpital, les fournisseurs de prestations disposent encore de certaines marges pour compenser les sous-financements dus à des structures tarifaires insuffisamment correctes. Toutefois, des taux de base négociables réduisent aussi la pression économique sur les fournisseurs de prestations, pression qui était un objectif majeur de l'introduction de SwissDRG au sens d'une mise en évidence dans les hôpitaux des réserves d'efficiences présumées par le législateur [3, $5,6]$. Pour parvenir à une sécurité de planification, il faut cependant en premier lieu trouver des solutions correctes au sein de la structure tarifaire. Dans la discussion sur les différents taux de base pour différents niveaux de soins, il faudrait tenir compte de l'influence sur la compétitivité des hôpitaux [3].

\section{Conclusion}

Le système SwissDRG poursuit son développement au sens d'un «système d'apprentissage». La version 2.0 améliore, dans la limite des possibilités existantes, la justesse de la prise en compte des prestations par rapport à la version précédente [4]; elle repose sur une base améliorée, constituée de données sur les prestations (diagnostics et procédures plus différenciés) et sur les coûts (liste unique des médicaments, notamment). Cette base sera encore améliorée par des directives plus fermes et ciblées (voir plus haut). Dans ce contexte, la poursuite de la collabora- tion entre l'InEK et SwissDRG SA apparaît très utile. La réduction du nombre de DRG, c'est-à-dire la condensation de la structure tarifaire, concerne des domaines qui ont été structurés de façon déterminante par des données allemandes helvétisées. Les nombres réduits de cas, les faiblesses de la calculation des coûts et, en partie, les structures de soins différentes rendent indispensables des solutions suisses à part entière. Ce processus a déjà commencé et va se poursuivre.

Comme on pouvait s'y attendre, la version 2.0 ne peut constituer qu'une étape intermédiaire sur la voie menant aux objectifs fixés par le législateur suisse pour l'introduction du système SwissDRG. Tant au sein de la structure tarifaire qu'en dehors de celle-ci, il faudra entreprendre d'autres étapes importantes afin de parvenir à une calculation correcte des coûts par cas sur la base de données suisses sur les prestations et les coûts, ainsi qu'à une qualité élevée de leur prise en compte par le système SwissDRG. La prochaine version (3.0) se fondera notamment sur des changements majeurs des structures CIM-10-GM et CHOP, si bien qu'on pourra s'attendre à l'avenir à une nouvelle augmentation de la justesse de prise en compte des prestations.

\section{Références}

1 SwissDRG SA: Catalogue des forfaits par cas SwissDRG version 1.0. A télécharger sur: www.swissdrg.org/fr/07 casemix_office/SwissDRG_System_10.asp?navid=29

2 SwissDRG SA: Catalogue des forfaits par cas SwissDRG version 2.0. A télécharger sur: www.swissdrg.org/fr/07 casemix_office/SwissDRG_System_20.asp?navid=25

3 Meyer B, Ingenpass P SwissDRG-Version 2.0 - avis de la FMH et recommandations. Bull Méd Suisses. 2012; 93(22):797-8.

4 SwissDRG SA: diapositives de la présentation SwissDRG (en allemand) «SwissDRG-Version 2.0/2013», Berne 13.6.12. A télécharger sur: www.swissdrg.org/de/06_swissdrg_ag/Veranstaltungen/20120613_Version_2.0_Homepage.pdf

5 Franz D, Bunzemeier H, Fiori W, Wenke A, Helling J, Brüning K et al. Die SwissDRGs - ein Zwischenbericht zur Einführung der fallpauschalierten Spitalfinanzierung auf der Grundlage deutscher DRG-Strukturen (I). Das Krankenhaus. 2012; 11:1074-80.

6 Franz D, Bunzemeier H, Fiori W, Wenke A, Helling J, Brüning K, et al. Die SwissDRGs - ein Zwischenbericht zur Einführung der fallpauschalierten Spitalfinanzierung auf der Grundlage deutscher DRG-Strukturen (II). Das Krankenhaus. 2010;12:1196-1204.

7 SwissDRG SA: Mise en œuvre des mesures d'amélioration de la qualité des données. Version 1.0 / 5.12.2011. A télécharger sur: www.swissdrg.org/assets/pdf/ Erhebung2012/Umsetzung_Massnahmen_Daten qualitaet-f_aw.pdf

8 REKOLE - comptabilité de gestion à l'hôpital. H+ - Les hôpitaux de Suisse (éditeur) ISBN 978-3-9523416-1-2. 